\title{
Predictive value of maternal serum podocalyxin in the diagnosis of preeclampsia: a prospective case-control study
}

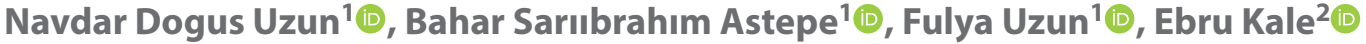 \\ ${ }^{1}$ Department of Obstetrics and Gynecology, Health Sciences University Derince Training And Research Hospital, Kocaeli, Turkey \\ ${ }^{2}$ Department of Biochemistry, Doctor Lufti Kirdar Kartal Training and Research Hospital, Istanbul, Turkey
}

\begin{abstract}
Objectives: There is a need for markers to facilitate the diagnosis of preeclampsia, one of the most chief causes of maternal and infant mortality. Preeclampsia causes damage to the glomeruli and vascular endothelium in pregnant women. Podocalyxin is a sialoglycoprotein found in both glomeruli and vascular endothelium. In this study, we investigated the levels of podocalyxin in preeclampsia, and studied its potential to predict preeclampsia.

Material and methods: Women admitted to the Health Sciences University Derince Training and Research Hospital, Department of Obstetrics and Gynecology between February-November 2018 due to high direct blood and diagnosed with preeclampsia according to the 2013 American College of Obstetricians and Gynecologists criteria were included in the study. The control group consisted of healthy volunteers having similar demographic features (gestational week, gravida, parity, and age) with the preeclampsia group. The main outcome variable was serum podocalyxin levels.

Results: The mean $( \pm$ SD) podocalyxin levels of the study and control groups were $124.15 \pm 39.63 \mathrm{ng} / \mathrm{mL}$ and $71.47 \pm 16.86 \mathrm{ng} / \mathrm{mL}$, respectively $(\mathrm{t}=7.845, \mathrm{p}<0.001)$. Using a cut-off of 91.7123 , podocalyxin could predict preeclampsia with $90 \%$ sensitivity and $98 \%$ specificity. Furthermore, podocalyxin levels were significantly higher than the normotensive participants in both early $(143.81 \pm 51.96 \mathrm{ng} / \mathrm{mL}$ vs $75.35 \pm 19.36 \mathrm{ng} / \mathrm{mL})$ and late-onset $(110.22 \pm 19.11 \mathrm{ng} / \mathrm{mL}$ vs $68.26 \pm 14.13 \mathrm{ng} / \mathrm{mL})$ preeclampsia $(p<0.001)$.
\end{abstract}

Conclusions: Serum podocalyxin levels increase in preeclampsia. We conclude that podocalyxin is a candidate for predicting preeclampsia.

Key words: early diagnosis; maternal serum; podocalyxin; preeclampsia

\section{INTRODUCTION}

\section{Background/rationale}

Preeclampsia is a systemic disorder that may affect both the mother and the fetus. It can cause serious cardiorespiratory, neurologic, renal, hepatic, and hematologic complications [1]. Preeclampsia is the second most common cause of maternal mortality in Turkey [2]. According to the World Health Report 2015, approximately 830 women die every day due to complications ensuing during pregnancy or delivery; the number of women died in 2016 was reported as 303,000 [3].

Preeclampsia increases fetal risks associated with stillbirth, neonatal death, intrauterine growth retardation, and premature birth [4]. In addition, it has been implicated in increasing post-partum hypertension and chronic kidney disease [5].

Preeclampsia directly damages the glomerular endothelium, consequently causing acute renal injury. Thus, angiogenic instability is a trigger factor for the damage of both podocytes and the endothelium in preeclampsia [6].

On the other hand, podocalyxin is a glomerular podocyte protein, also secreted from endothelial cells of other organs, which increases in the urine of preeclamptic women [7].

Some studies have reported that podocyturia may be used to predict preeclampsia and determine its severity [8-10]. However, a recent study indicated podocalyxin in pregnant women could be detected with the ELISA kit and 
speculated it could be used as a predictive tool for early onset preeclampsia [7].

\section{Objectives}

This study investigated whether podocalyxin can be used as a predictive tool in preeclampsia.

\section{MATERIAL AND METHODS Study design}

This study was designed as a prospective case-control study. Study reporting was done per the STROBE guideline [11]. Written informed permission was obtained from all participants. The study protocol was approved by the Local Ethical Committee of Non-Invasive Clinical Research at Kocaeli University Research Hospital (IRB number: 2018/54; Date: 07.02.2018).

\section{Setting}

This research was carried out in Kocaeli Obstetrics and Gynecology Department of Derince Training and Research Hospital between February-November 2018.

\section{Participants}

Participants of the study consisted of 41 preeclamptic and 42 healthy pregnant women. Seventeen early-onset preeclampsia patients and 24 late-onset preeclampsia patients were included in the study at our clinic during the study period. The preeclamptic group included patients applied to the obstetrics and gynecology emergency department of Health Sciences University Derince Training and Research Hospital. Preeclampsia patients were chosen according to the 2013 American College of Obstetricians and Gynecologists (ACOG) criteria. According to ACOG: Preeclampsia is defined as hypertension combined with proteinuria, or in absence of proteinuria, combined with at least one or more other findings including maternal organ dysfunction (elevated liver enzymes, haematological complications, renal insufficiency, neurological symptoms) and pulmonary edema. Hypertension is classified either as new onset hypertension after 20 weeks of gestation with blood pressure levels $\geq 140 / 90 \mathrm{mmHg}$ on two occasions at least $4 \mathrm{~h}$ apart, or as chronic hypertension. Severe features of preeclampsia include blood pressure at least $\geq 160 / 110 \mathrm{~mm} \mathrm{Hg}$, platelet count less than $100 \times 103$ per $\mu \mathrm{L}$, liver transaminase levels two times the upper limit of normal, a doubling of the serum creatinine level or level greater than $1.1 \mathrm{mg}$ per $\mathrm{dL}$, severe persistent right upper-quadrant pain, pulmonary edema, or new-onset cerebral or visual disturbances. Normotensive healthy pregnant volunteers with similar gestational week, gravida, parity, and age as in the preeclampsia group constituted the control group. The control participants were pregnant women seen in the same center during the study period who had no high blood pressure during the follow-up, did not have any systemic disease, and did not use any drugs except vitamin and iron supplementations. All participants were at or beyond the $20^{\text {th }}$ gestational week. Patients who had previously high blood pressure, renal or liver disease, intermittent hypertension, or proteinuria before pregnancy were excluded from the study (Fig. 1). Detailed physical examination and routine blood tests were ordered in all patients. Patients were informed by the attending physician, and if approved, $5 \mathrm{~mL}$ blood was taken in addition to routine blood tests. Preeclampsia was diagnosed according to the 2013 American College of Obstetricians and Gynecologists (ACOG) criteria.

\section{Variables}

The primary outcome variable of the study was "serum podocalyxin level". Secondary outcome variables were routinely ordered tests including serum alanine transaminase (ALT), aspartate transaminase (AST), lactate dehydrogenase (LDH), urea, creatinine, platelet count, urine protein/creatinine ratio, and 24-hour urine protein excretion. The routine tests were analyzed in the biochemistry laboratory of the hospital per hospital protocol. The blood obtained for podocalyxin analysis was centrifuged at $2500 \mathrm{rpm}$ for 10 minutes within 30 minutes after

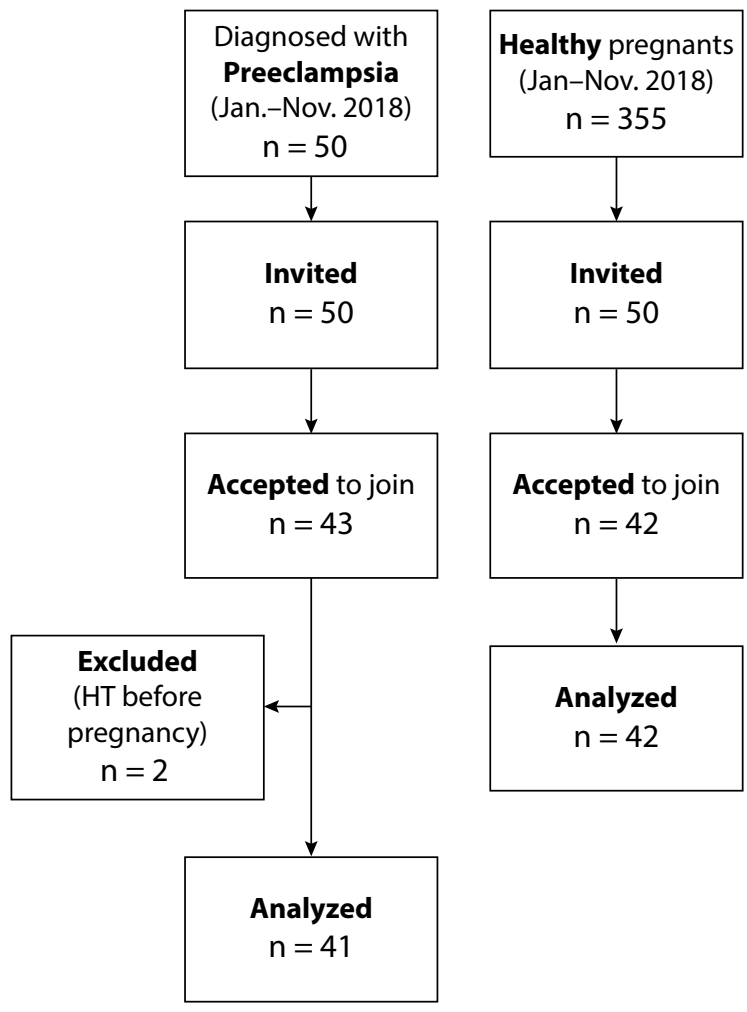

Figure 1. Participant flow diagram; HT — hypertension 
collection. The sera obtained were stored at $-80^{\circ} \mathrm{C}$ until the time of analysis. Podocalyxin was studied using ELISA (Elabscience ${ }^{\bullet}$, Hubei/China). Studies were carried out in accordance with the kit protocol. The ELISA kit used works with the competitive ELISA method.

\section{Study size}

The sample size was calculated based on the primary outcome variable with a minimum of $80 \%$ power and a maximum of $5 \%$ type 1 error to find a statistically significant difference between the study groups. The calculation with the power analysis of the E-picos section of the Medicres program revealed 37 participants in each group for a $95 \%$ confidence interval. Serum podocalyxin levels were assumed as $50 \pm 12 \mathrm{ng} / \mathrm{mL}$ and $60 \pm 12 \mathrm{ng} / \mathrm{mL}$ for normotensive healthy pregnant women and the preeclampsia group, respectively.

\section{Statistical analysis}

The data were analyzed using the Statistical Package for the Social Sciences (SPSS) version 25.0 software (SPSS Inc., Chicago, IL, USA). The results of the study were presented as frequencies and percentages for categorical variables and as means and standard deviations for numerical variables. The normal distribution of the numerical variables was evaluated by checking the skewness coefficients. The independent samples t-test, Mann-Whitney U test, or one-way ANOVA were used to compare the groups in cases where parametric test conditions were met. Post hoc analyzes were performed with Tukey if the variances were homogeneous and Tamhane T2 if not. The receiver operating characteristics (ROC) analysis was used to determine sensitivity and specificity values for podocalyxin. Multivariate comparisons were examined by logistic regression analysis and two-way ANOVA. The statistical significance threshold was taken as $p<0.05$.

\section{RESULTS}

Data for 83 participants were analyzed. Forty-one of them were preeclamptic, and forty-two were healthy pregnant. The mean age of the participants was $28.46 \pm 5.28$ years and range were between 18-40 (Tab. 1).

According to urine dipstick results, 7 patients did not show proteinuria, 5 patients had trace proteinuria, 6 patients had +1 proteinuria, 13 patients had +2 proteinuria and 10 patients had +3 proteinuria. When podocalyxin levels were compared with the protein status in the urine, no statistically significant difference was detected ( $p=0.417$ ).

Podocalyxin, ALT, AST, LDH, urea, creatinine, systolic blood pressure, and diastolic blood pressure were significantly different in the preeclamptic (case) group compared to the controls. However, therewasnosignificant differenceintheurineprotein/creatinine ratio and 24-hour urine protein excretion (Tab. 2).

\begin{tabular}{|c|c|c|c|c|c|}
\hline & $n$ & Mean & SD & Minimum & Maximum \\
\hline Age [year] & 83 & 28.46 & 5.28 & 18 & 40 \\
\hline Height [cm] & 83 & 160.90 & 6.87 & 148 & 175 \\
\hline Weight [kg] & 83 & 86.65 & 16.62 & 57 & 130 \\
\hline $\begin{array}{l}\text { Hemoglobin } \\
{[\mathrm{mg} / \mathrm{dL}]}\end{array}$ & 83 & 11.37 & 1.26 & 8.40 & 14.90 \\
\hline Hematocrit [\%] & 83 & 34.84 & 3.49 & 27.00 & 43.30 \\
\hline $\begin{array}{l}\text { Platelets } \\
\text { [number/mL] }\end{array}$ & 83 & 202.42 & 60.11 & 27 & 352 \\
\hline ALT & 83 & 19.55 & 35.53 & 6 & 234 \\
\hline AST & 83 & 23.67 & 23.74 & 10 & 173 \\
\hline LDH & 83 & 241.27 & 88.85 & 120 & 617 \\
\hline Creatinine & 83 & 0.56 & 0.07 & 0.43 & 0.88 \\
\hline
\end{tabular}

SD — standard deviation; ALT — alanine transaminase; AST — aspartate transaminase; $\mathrm{LDH}$ - lactate dehydrogenase

Also, patients with early preeclampsia had significantly higher mean podocalyxin levels compared to those with late onset ( $143.81 \pm 51.96 \mathrm{ng} / \mathrm{mL}$ vs. $110.22 \pm 19.11 \mathrm{ng} / \mathrm{mL}$ ) (Mann-Whitney U Z = 2.435; $\mathrm{p}=0.015$ ).

The ROC analysis demonstrated that podocalyxin provides a significant advantage in predicting preeclampsia (Area under the curve $0.939 \mathrm{p}<0.001$ ) (Fig. 2). A podocalyxin cut-off level of 91.71 provides $90 \%$ sensitivity and $98 \%$ specificity in foreseeing preeclampsia.

Podocalyxin showed significant positive correlations with urea, creatinine, ALT, AST, and LDH ( $r$ p $0.417 ;<0.001$, $0.372 ; 0.001,0.226 ; 0.040,0.327 ; 0.003$, and $0.353 ; 0.001$, respectively), and a significant negative correlation with platelet count $(r=-0.373 ; p=0.001)$.

A logistic regression analysis with preeclampsia status as the dependent and podocalyxin, and urea, creatinine, ALT, AST, LDH, and platelet levels as independent variables, demonstrated that podocalyxin was the only significant independent predictor of preeclampsia status (Wald = 15.951, $\mathrm{p}<0.001, \operatorname{Exp}(\mathrm{B})=1.153,95 \% \mathrm{Cl}: 1.075-1.236)$

\section{DISCUSSION}

Key results

This study demonstrated that serum podocolyxin levels are increased in preeclamptic pregnancies. Cases with early onset had significantly higher podocalyxin levels compared with late onset.

\section{Limitations}

One limitation of this is the lack of Podocolyxin information of the participants before their pregnancies. Large-scale cohort studies are needed to calculate the odds of baseline podocalyxin levels in predicting preeclampsia. 
Table 2. Comparison of the preeclamptic and control groups concerning the outcome measures

\begin{tabular}{|c|c|c|c|c|c|c|}
\hline & Group & $\mathbf{N}$ & Mean & SD & *p & $\mathbf{t}$ \\
\hline \multirow{2}{*}{ Podocalyxin [ng/mL] } & Preeclamptic & 41 & 124.15 & 39.63 & $<0.001$ & 7.845 \\
\hline & Control & 42 & 71.47 & 16.86 & & \\
\hline \multirow{2}{*}{ Systolic blood pressure [mmHg] } & Preeclamptic & 41 & 151.71 & 12.82 & $<0.001$ & 17.769 \\
\hline & Control & 42 & 105.71 & 10.68 & & \\
\hline \multirow{2}{*}{ Diastolic blood pressure [mmHg] } & Preeclamptic & 41 & 98.54 & 6.54 & $<0.001$ & 17.724 \\
\hline & Control & 42 & 68.69 & 8.62 & & \\
\hline \multirow{2}{*}{ Urine protein/creatinine ratio } & Preeclamptic & 21 & 2.43 & 4.13 & 0.054 & 2.036 \\
\hline & Control & 7 & 0.52 & 0.66 & & \\
\hline \multirow{2}{*}{ 24-hour urine protein excretion [mg] } & Preeclamptic & 8 & 2306.85 & 4367.97 & 0.529 & 0.658 \\
\hline & Control & 2 & 181.50 & 86.97 & & \\
\hline \multirow{2}{*}{ Platelets [number/mL] } & Preeclamptic & 41 & 182.98 & 62.25 & 0.003 & -3.056 \\
\hline & Control & 42 & 221.40 & 51.95 & & \\
\hline \multirow{2}{*}{ ALT } & Preeclamptic & 41 & 28.83 & 48.88 & 0.018 & 2.389 \\
\hline & Control & 42 & 10.50 & 4.92 & & \\
\hline \multirow{2}{*}{ AST } & Preeclamptic & 41 & 31.73 & 31.63 & 0.003 & 3.187 \\
\hline & Control & 42 & 15.81 & 4.81 & & \\
\hline \multirow{2}{*}{ LDH } & Preeclamptic & 41 & 282.20 & 100.14 & $<0.001$ & 4.603 \\
\hline & Control & 42 & 201.31 & 51.92 & & \\
\hline \multirow{2}{*}{ Creatinine } & Preeclamptic & 41 & 0.58 & 0.09 & 0.027 & 2.255 \\
\hline & Control & 42 & 0.55 & 0.06 & & \\
\hline \multirow{2}{*}{ Urea } & Preeclamptic & 41 & 18.88 & 6.85 & 0.005 & 2.935 \\
\hline & Control & 42 & 15.12 & 4.55 & & \\
\hline
\end{tabular}

*Independent samples t-test; SD — standard deviation; ALT — alanine transaminase; AST — aspartate transaminase; LDH — lactate dehydrogenase

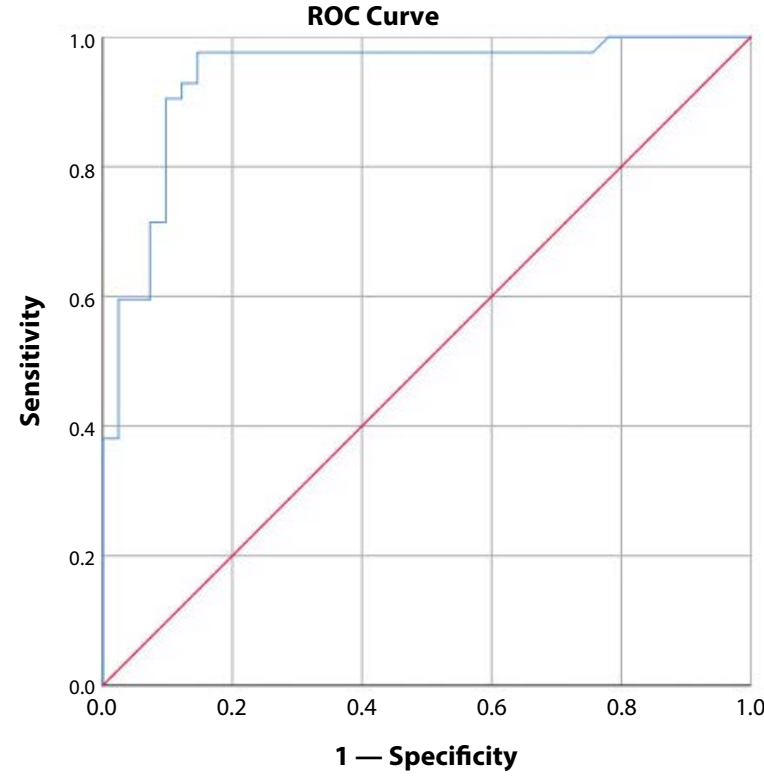

Diagonal segments are produced by ties

Figure 2. Receiver operating characteristics graph showing podocalyxin levels in predicting preeclampsia; $\mathrm{ROC}$ - receiver operating characteristics

\section{Interpretation}

The diagnosis of preeclampsia, one of the most prominent causes of maternal and fetal morbidity and mortality, affecting $3-7 \%$ of healthy nulliparous and $1-3 \%$ of multiparous women, is of vital importance [12]. It was suggested that podocyturia screening at the end of the second trimester could identify pregnant women at risk for preeclampsia [13]. A study conducted in 2017, stated that serum podocalyxin values were higher in early preeclamptic pregnant women compared to a control group [7]. Our findings support this result and further add that the podocalyxin levels are significantly higher also in late-onset preeclampsia.

In other words, all preeclamptic pregnant women had higher podocalyxin levels. This finding is not surprising. Because the pathogenesis of preeclampsia, such as incomplete spiral artery remodeling that contribute to placental ischemia and release of antiangiogenic factors from the ischemic placenta to the maternal circulation causing endothelial damage, also affect podocalyxin levels. Additionally, the glomerular endothelium is directly damaged in preeclampsia, and podocalyxin is abundant in the renal glomeruli [14]. Podocalyxin is a glomerular podocyte protein, but it is secreted 
from endothelial cells of other organs too. We postulate that podocalyxin secreted from maternal endothelial cells may increase in the sera of preeclamptic women.

Preeclampsia occurs in 2-5\% of pregnancies in developed countries. However, it may complicate up to $10 \%$ of pregnancies in developing countries, where emergency care may not be adequate [15]. In 2004, after conducting a systematic review of screening tests for preeclampsia, the World Health Organization reported that there was no clinically useful screening test to predict the development of preeclampsia in low-risk or high-risk populations, and advised for further studies [16]. After this report, many researchers have identified or examined potential biochemical and/or biophysical markers. Some systematic reviews and meta-analyzes evaluating the clinical benefits of studies with a single marker have been published [17-19]. However, the need for a suitable marker getting a high level of accuracy persisted [20].

To be effective, a screening test must be sufficiently sensitive and specific and provide an adequate positive predictive value. The argument that podocalyxin values can be used as a predictor in preeclampsia was noted as one of the essential findings of this study. For the first time serum podocalyxin was found to be successful in predicting preeclampsia at $90 \%$ sensitivity and $98 \%$ specificity. We want to speculate that the sensitivity and specificity of podocalyxin are high enough to suggest its involvement in preeclampsia diagnosis.

Although there are some conflicting studies, report generally support higher liver function tests in preeclamptic pregnant women [21-23]. According to a recent study, elevated AST and ALT levels in the first 20 weeks of pregnancy are associated with a higher risk of developing severe preeclampsia in the second half of the pregnancy. However, there is no clinical cut-off value that can be used practically to predict preeclampsia [24].

In a study consisting of preeclampsia, severe preeclampsia, and control groups, hemoglobin values were lower in the patients with severe preeclampsia. However, ALT, AST, urea, and creatinine values too were significantly higher in this group [25]. In our study, no difference was found between the groups concerning hemoglobin values. In the preeclampsia group, ALT, AST, LDH, urea, and creatinine levels were significantly different from the control group, which was coherent with previous studies and expectations. However, it was surprising that there was no statistically significant difference in the urine protein/creatinine ratio and 24-hour urine protein excretion. This result was thought to be due to the low number of data on protein excretion in 24-hour urine.

In our study, preeclampsia was divided into two groups as early and late according to the time of onset. There was no statistically significant difference in other variables ex- cept podocalyxin in these two groups. This finding suggested that more focus should be placed on podocalyxin to elucidate the pathogenesis of preeclampsia. The remarkable point was that the level of podocalyxin was lower in late-onset preeclampsia than in the early-onset cases. In addition, there was no significant difference between severe preeclampsia and preeclampsia groups regarding podocalyxin levels. This suggests that podocalyxin is elevated independently of hypertension in preeclampsia. However, the low number of severe preeclampsia groups indicates that this result should be supported by larger studies. Probably endothelial damage is required to increase podocalyxin levels. Once damage occurs, its severity may not further increase podocalyxin levels.

Lactate dehydrogenase, the key enzyme of glycolysis, is used to identify the cause and location of tissue damage in the body and to help monitor the progress of the damage. LDH increases in many diseases as a result of its widespread distribution in the tissues [26]. On the other hand, podocalyxin has been reported to be a marker of embryonic hematopoietic stem cells (HSCs), erythroid cells and adult $\mathrm{HSCs}$, and thus, may be a valuable marker for purification of these cells for transplantation [27]. It was suggested that the correlation between LDH and podocalyxin and the similarly their high levels in preeclamptic pregnant women can be attributed to the damage caused by preeclampsia. The correlation between $\mathrm{LDH}$ and podocalyxin, and heir surge in preeclamptic pregnant women may be due to the damage to tissues where both markers are dense.

\section{CONCLUSIONS}

Serum podocalyxin levels are increased in preeclamptic pregnant women. The serum podocalyxin levels are higher in early onset preeclampsia compared to late onset cases. However, the severity of preeclampsia does not make a significant difference. We conclude that with $90 \%$ sensitivity and $98 \%$ specificity, podocalyxin is a candidate for predicting preeclampsia.

\section{Funding}

This study was not funded by any organization.

\section{Conflict of interest}

The authors have no conflict of interest in this study.

\section{REFERENCES}

1. Bramham K, Parnell B, Nelson-Piercy C, et al. Chronic hypertension and pregnancy outcomes: systematic review and meta-analysis. BMJ. 2014; 348: g2301, doi: 10.1136/bmj.g2301, indexed in Pubmed: 24735917.

2. Köse MR, Bora Başara B, Güler C. Health Statistics Yearly 2016 News Bulletin.Bora Başara B, Güler C, Soytutan Çağlar I. ed. Sağlık Araştırmaları Genel Müdürlüğü, Ankara 2017.

3. Merdad L, Ali MM. Timing of maternal death: Levels, trends, and ecological correlates using sibling data from 34 sub-Saharan Afri- 
can countries. PLoS One. 2018; 13(1): e0189416, doi: 10.1371/journal. pone.0189416, indexed in Pubmed: 29342157.

4. Hutcheon JA, Lisonkova S, Joseph KS. Epidemiology of pre-eclampsia and the other hypertensive disorders of pregnancy. Best Pract Res Clin Obstet Gynaecol. 2011; 25(4): 391-403, doi: 10.1016/j.bpobgyn.2011.01.006, indexed in Pubmed: 21333604.

5. Turbeville HR, Taylor EB, Garrett MR, et al. Superimposed Preeclampsia Exacerbates Postpartum Renal Injury Despite Lack of Long-Term Blood Pressure Difference in the Dahl Salt-Sensitive Rat. Hypertension. 2019; 73(3):650-658, doi: 10.1161/HYPERTENSIONAHA.118.12097, indexed in Pubmed:30612494.

6. Turner RJ, Bloemenkamp KWM, Penning ME, et al. From Glomerular Endothelium to Podocyte Pathobiology in Preeclampsia: a Paradigm Shift. Curr Hypertens Rep. 2015; 17(7): 54, doi: 10.1007/s11906-015-0566-9, indexed in Pubmed: 26068658.

7. Chen Qi, Wang Y, Li Y, et al. Serum podocalyxin is significantly increased in early-onset preeclampsia and may represent a novel marker of maternal endothelial cell dysfunction. J Hypertens. 2017; 35(11): 2287-2294, doi: 10.1097/HJH.0000000000001461, indexed in Pubmed: 28665887.

8. Panek-Laszczyńska K, Konieczny A, Milewska E, et al. Podocyturia as an early diagnostic marker of preeclampsia: a literature review. Biomarkers. 2018;23(3): 207-212, doi: 10.1080/1354750X.2017.1405283, indexed in Pubmed: 29130350.

9. Jim B, Mehta S, Qipo A, et al. A comparison of podocyturia, albuminuria and nephrinuria in predicting the development of preeclampsia: a prospective study. PLoS One. 2014; 9(7): e101445, doi: 10.1371/journal. pone.0101445, indexed in Pubmed: 25010746.

10. Kelder TP, Penning ME, Uh HW, et al. Quantitative polymerase chain reaction-based analysis of podocyturia is a feasible diagnostic tool in preeclampsia. Hypertension. 2012; 60(6): 1538-1544, doi: 10.1161/HYPERTENSIONAHA.112.201681, indexed in Pubmed: 23090771.

11. von Elm E, Altman DG, Egger M, et al. STROBE Initiative. The Strengthening the Reporting of Observational Studies in Epidemiology (STROBE) statement: guidelines for reporting observational studies. J Clin Epidemiol. 2008; 61(4): 344-349, doi: 10.1016/j.jclinepi.2007.11.008, indexed in Pubmed: 18313558.

12. Hernández-Díaz S, Toh S, Cnattingius S. Risk of pre-eclampsia in first and subsequent pregnancies: prospective cohort study. BMJ. 2009; 338: b2255, doi: 10.1136/bmj.b2255, indexed in Pubmed: 19541696.

13. Craici IM, Wagner SJ, Bailey KR, et al. Podocyturia predates proteinuria and clinical features of preeclampsia: longitudinal prospective study. Hypertension. 2013; 61(6): 1289-1296, doi: 10.1161/HYPERTENSIONAHA.113.01115, indexed in Pubmed: 23529165.

14. Montesano R, Matsumoto $K$, Nakamura T, et al. Identification of a fibroblast-derived epithelial morphogen as hepatocyte growth factor. Cell. 1991; 67(5): 901-908, doi: 10.1016/0092-8674(91)90363-4, indexed in Pubmed: 1835669.
15. Monte S. Biochemical markers for prediction of preclampsia: review of the literature. J Prenat Med. 2011; 5(3): 69-77, indexed in Pubmed: 22439080.

16. Conde-Agudelo A, Villar J, Lindheimer M. World Health Organization systematic review of screening tests for preeclampsia. Obstet Gynecol. 2004; 104(6): 1367-1391, doi: 10.1097/01.AOG.0000147599.47713.5d, indexed in Pubmed: 15572504.

17. Thangaratinam S, Coomarasamy A, Sharp S, et al. Tests for predicting complications of pre-eclampsia: a protocol for systematic reviews. BMC Pregnancy Childbirth. 2008; 8: 38, doi: 10.1186/1471-2393-8-38, indexed in Pubmed: 18694494.

18. Cnossen JS, van der Post JAM, Mol BWJ, et al. Prediction of pre-eclampsia: a protocol for systematic reviews of test accuracy. BMC Pregnancy Childbirth. 2006; 6: 29, doi: 10.1186/1471-2393-6-29, indexed in Pubmed: 17052339.

19. Wang D, Cummins C, Bayliss S, et al. Immunoprophylaxis against respiratory syncytial virus (RSV) with palivizumab in children: a systematic review and economic evaluation. Health Technol Assess. 2008; 12(36): iii, ix-x, 1, doi: 10.3310/hta12360, indexed in Pubmed: 19049692.

20. Giguère $Y, C$ harland $M, B$ ujold $E$, et al. Combining biochemical and ultrasonographic markers in predicting preeclampsia: a systematic review. Clin Chem. 2010; 56(3): 361-375, doi: 10.1373/clinchem.2009.134080, indexed in Pubmed: 20044446.

21. El-Sayed AAF. Preeclampsia: A review of the pathogenesis and possible management strategies based on its pathophysiological derangements. Taiwan J Obstet Gynecol. 2017; 56(5): 593-598, doi: 10.1016/j. tjog.2017.08.004, indexed in Pubmed: 29037542.

22. Sibai BM, Taslimi MM, el-Nazer A, et al. Maternal-perinatal outcome associated with the syndrome of hemolysis, elevated liver enzymes, and low platelets in severe preeclampsia-eclampsia. Am J Obstet Gynecol. 1986; 155(3): 501-509, doi: 10.1016/0002-9378(86)90266-8, indexed in Pubmed: 3529964.

23. Makuyana D, Mahomed K, Shukusho FD, et al. Liver and kidney function tests in normal and pre-eclamptic gestation--a comparison with non-gestational reference values. Cent Afr J Med. 2002; 48(5-6): 55-59.

24. Büyükbaş S, İnal A. Changes in Serum Lactate Dehydrogenase Isoenzymes in Moderate Drinkers]. Van Med J. 2006; 13(3): 85-89.

25. Taşın C, Yıldız Y, Ünlü Bekir S, et al. Evaluation of Maternal and Perinatal Findings in Mild and Severe Preeclampsia Cases]. Kocatepe Tıp Derg Kocatepe Med J. 2014; 15(1): 7-12.

26. Augoff K, Grabowski K. [Significance of lactate dehydrogenase measurements in diagnosis of malignancies]. Pol Merkur Lekarski. 2004; 17(102): 644-647, indexed in Pubmed: 15771142.

27. Doyonnas R, Nielsen JS, Chelliah S, et al. Podocalyxin is a CD34-related marker of murine hematopoietic stem cells and embryonic erythroid cells. Blood. 2005; 105(11):4170-4178, doi: 10.1182/blood-2004-10-4077, indexed in Pubmed: 15701716. 